\title{
Diagnostic value of single complete compression ultrasonography in pregnant and postpartum women with suspected deep vein thrombosis: prospective study \\ (c)
}

Grégoire Le Gal professor of internal medicine ${ }^{12}$, Geneviève Kercret vascular medicine physician ${ }^{3}$, Khalil Ben Yahmed associate physician of internal medicine ${ }^{2}$, Luc Bressollette professor of vascular medicine $^{2}$, Helia Robert-Ebadi associate physician of angiology ${ }^{4}$, Louise Riberdy research nurse ${ }^{4}$, Patrick Louis vascular medicine physician ${ }^{5}$, Aurélien Delluc assistant professor of internal medicine ${ }^{2}$, Marie-Luce Labalette vascular medicine physician ${ }^{6}$, Mohamed Baba-Ahmed vascular medicine physician ${ }^{2}$, Henri Bounameaux professor of angiology ${ }^{4}$, Dominique Mottier professor of therapeutics $^{12}$, Marc Righini angiology physician ${ }^{4}$, on behalf of the EDVIGE study group

${ }^{1}$ INSERM CIC 05-02, Centre Hospitalier Universitaire de la Cavale Blanche, Brest, France; ${ }^{2}$ Département de médecine interne et de pneumologie, Centre Hospitalier Universitaire de la Cavale Blanche, Boulevard Tanguy Prigent, 29609 Brest, France; ${ }^{3} 3$, rue Gambetta, Rennes, France; ${ }^{4}$ Division of Angiology and Hemostasis, Geneva University Hospital, Geneva, Switzerland; ${ }^{2}$, rue Farigoul, Brest, France; ${ }^{6} 21$, rue Saint-Nicolas, Lannion, France

\begin{abstract}
Objective To assess the safety of using single complete compression ultrasonography in pregnant and postpartum women to rule out deep vein thrombosis.

Design Prospective outcome study.

Setting Two tertiary care centres and 18 private practices specialising in vascular medicine in France and Switzerland.

Participants 226 pregnant and postpartum women referred for suspected deep vein thrombosis.

Methods A single proximal and distal compression ultrasonography was performed. All women with a negative complete compression ultrasonography result did not receive anticoagulant therapy and were followed up for a three month period.
\end{abstract}

Main outcome measures Symptoms of venous thromboembolism, second compression ultrasonography or chest imaging, a thromboembolic event, and anticoagulant treatment.

Results 16 women were excluded, mainly because of associated suspected pulmonary embolism. Deep vein thrombosis was diagnosed in 22 out of the 210 included women (10.5\%). 10 patients received full dose anticoagulation despite a negative test result during follow-up. Of the 177 patients without deep vein thrombosis and who did not receive full dose anticoagulant therapy, two (1.1\%, $95 \%$ confidence interval $0.3 \%$ to $4.0 \%$ ) had an objectively confirmed deep vein thrombosis during follow-up.

Conclusions The rate of venous thromboembolic events after single complete compression ultrasonography in pregnant and postpartum women seems to be within the range of that observed in studies in the non-pregnant population. These data suggest that a negative single complete compression ultrasonography result may safely exclude the diagnosis of deep vein thrombosis in this setting.

Trial registration clinicaltrials.gov NCT00740454.

\section{Introduction}

Because pregnancy is known to be a risk factor for venous thromboembolism and pregnant women often experience symptoms compatible with deep vein thrombosis (pain, tenderness, and swelling of the legs), the threshold for clinical suspicion and the accuracy of clinical examination are reduced. ${ }^{12}$ Misdiagnoses during pregnancy, however, should be avoided as much as possible - for example, false positive test results lead to inappropriate anticoagulant treatment, which increases the risk of bleeding and requires daily heparin injections during the pregnancy. In contrast, false negative test results may lead to a life threatening thromboembolic event. Thus the accuracy of diagnostic methods used in pregnant women is crucial. 
Phlebography remains the reference method for the diagnosis of deep vein thrombosis, ${ }^{3}$ but it is invasive and costly and exposes the fetus to radiation. Serial impedance plethysmography is the only non-invasive test that has been formally proved, in a prospective study, to be safe during pregnancy. ${ }^{1}$ This test is no longer in use, however, mainly because it has been shown to be less accurate than compression ultrasonography in non-pregnant patients. ${ }^{4}$ In such patients, assessment of clinical probability combined with D-dimer measurement is a well validated step for ruling out deep venous thrombosis. D-dimer levels, however, tend to increase steadily with pregnancy stage and to decrease slowly during the first weeks after delivery. ${ }^{5}$ As a result, the clinical usefulness - that is, the proportion of women with D-dimer levels below the diagnostic cut-off-is reduced. Moreover, the safety of ruling out deep vein thrombosis on the basis of D-dimer levels and clinical probability has never been prospectively validated in an outcome study of management during pregnancy.

In recent years, real time compression ultrasonography has become the main diagnostic test for suspected deep vein thrombosis. ${ }^{6}$ In non-pregnant patients with suspected deep vein thrombosis, a negative single complete compression ultrasonography result allows the diagnosis to be safely ruled out. ${ }^{7}$ Indeed, the rate of thromboembolic events in the three months after a negative single complete compression ultrasonography result has been shown to be about $1 \%$, a figure comparable to the $1.3 \%$ (95\% confidence interval $0.3 \%$ to $4.4 \%$ ) thromboembolic rate observed after a negative phlebography test result. ${ }^{38}$

Although compression ultrasonography is widely used, it has not been prospectively evaluated in pregnancy and post-partum. Its accuracy may be lower for several reasons. Firstly, pregnant women often present with conditions that make visualisation of the veins difficult, such as leg oedema or a gravid uterus, and that interfere with the visualisation of the proximal veins. Secondly, isolated iliac venous thromboses, which may be encountered more often in pregnancy, are more difficult to be diagnosed by compression ultrasonography, as the usual accepted criterion for deep vein thrombosis-lack of compressibility of the veins - may be difficult to evaluate at the iliac level in pregnant women. ${ }^{9}$ Thirdly, pregnancy is associated with changes in the anatomy and physiology of veins-namely, an increased vessel diameter and reduced flow velocity. ${ }^{10}$ These physiological changes are associated with technical difficulties for the ultrasound examination, and they persist for days or weeks after delivery.

Nevertheless, a retrospective study suggested that single complete compression ultrasonography may safely exclude deep vein thrombosis during pregnancy and post partum. ${ }^{11} \mathrm{We}$ assessed the safety of ruling out the diagnosis of deep vein thrombosis in pregnant or early postpartum women using single compression ultrasonography.

\section{Methods}

We included all pregnant or postpartum women referred with suspected deep vein thrombosis to two tertiary care centres and 18 private practices specialising in vascular medicine between January 2006 and June 2009. Pregnancy had to have been confirmed by the treating gynaecologist. Postpartum women were considered for inclusion until three months after delivery. Exclusion criteria were age less than 18 years, associated suspected pulmonary embolism, ongoing anticoagulant treatment, inability to give informed consent, and difficulties with follow-up.
Standardised report forms were filled in for all patients, recording general characteristics (age, date of delivery, end of pregnancy, height, weight, and weight gain) and risk factors for venous thromboembolism, including personal and family history of venous thromboembolic disease, recent surgery, trauma or immobilisation, history of varicose veins, complicated or twin pregnancy. Doctors were asked to empirically estimate the pretest clinical probability of deep vein thrombosis in three groups (low, intermediate, and high), although this had no impact on patient management.

\section{Venous ultrasonography}

We used high definition B mode ultrasound imaging equipment, with different probes according to the depth of the examined vessels. Iliac veins were visualised by direct imaging and Doppler flow. The whole venous network was scanned bilaterally: the inferior vena cava and iliac veins with the patient supine or in the contralateral position, femoral veins (common, superficial) and popliteal veins with the patient in a semi-upright position, and calf veins (posterior tibial and peroneal) with the patient in a sitting position and both feet resting on a chair. ${ }^{12} 13$ Study of the distal veins included the posterior tibial and peroneal veins, the gastrocnemius (internal and external), and the soleal veins, using different incidences. All of these venous segments were examined over their entire length in the transverse or longitudinal axis. The great and small saphenous veins were also studied at their junctions with the deep venous system. Special attention was paid to whether doctors were able to image all veins, in particular the ileocaval junction. All ultrasound examinations were performed by vascular medicine specialists with at least 10 years of experience in vascular ultrasound imaging.

\section{Diagnostic criteria}

We ruled out deep vein thrombosis in women with a negative compression ultrasonography test result and no visualised thrombus. Deep vein thrombosis was diagnosed with lack of compressibility of a deep vein and, for the iliac vein, in the absence of Doppler flow or direct visualisation of a thrombus.

\section{Follow-up}

We followed up all women with negative complete compression ultrasonography test results for a three month period. At the end of follow-up, the women were seen at the clinic or interviewed by telephone by the study staff using a standardised questionnaire to gather information about the three month period after compression ultrasonography: the women were asked if they had symptoms suggestive of venous thromboembolism, had undergone a second compression ultrasonography or chest imaging (computed tomography, ventilation-perfusion lung scan), had a diagnosis of deep vein thrombosis, or been treated with anticoagulant therapy. We excluded from analysis those women who received prolonged anticoagulant treatment during follow-up as this could bias the rate of recurrent deep vein thrombosis during follow-up. We also checked the participants' hospital medical records. When women could not be reached, a doctor from our research team called their general practitioner to complete follow-up. In case of a suspected event, we collected clinical notes and the results of diagnostic tests for adjudication. All suspected events were independently adjudicated by two expert doctors. A third doctor adjudicated discrepancies. 


\section{Statistical analysis}

The three month thromboembolic rate after a normal phlebography result in non-pregnant patients has been reported to be $1.3 \%$ (95\% confidence interval $0.3 \%$ to $4.4 \%$ ). We estimated that we had to obtain a complete follow-up in 180 women with a negative compression ultrasonography result to ensure that the upper limit of the $95 \%$ confidence interval was not higher than $4.0 \%$ around a $1.0 \%$ proportion of thromboembolic events during the three-month follow-up in women left untreated after a negative test result.

We computed the proportion of thromboembolic events during the three month period in women after a negative test result, along with $95 \%$ confidence intervals. Using a $\chi^{2}$ test or Fisher's exact test where applicable we estimated and tested the difference in the proportion of women with confirmed deep vein thrombosis according to stage of pregnancy, which leg had suspected deep vein thrombosis, and the clinical probability group.

\section{Results}

Overall, 226 pregnant or postpartum women with suspected deep vein thrombosis were included (figure $\Downarrow$ ). Sixteen were excluded: 14 because of a concomitant suspicion of pulmonary embolism and two because compression ultrasonography was performed as a routine test for follow-up of a previous deep vein thrombosis. Thus the final sample comprised 210 women. Table $1 \Downarrow$ shows the general characteristics of the women. The median age was 33 (interquartile range 28-37) years. Forty three women were in the postpartum period and 167 were pregnant: 20 in the first trimester, 51 in the second, and 96 in the third. In postpartum women, deep vein thrombosis was suspected after a median period of 5 (3-26) days. The pretest clinical probability was deemed low in $107(50.9 \%)$ women, intermediate in $85(40.5 \%)$, and high in $18(8.6 \%)$.

Deep vein thrombosis was diagnosed in 22 women. Of these, $20(91 \%)$ had a proximal (iliac, femoral, or popliteal) deep vein thrombosis, of whom four also had a distal deep vein thrombosis and one had a superficial vein thrombosis. The two remaining women had an isolated distal deep vein thrombosis. Thus the overall proportion of women with confirmed deep vein thrombosis was $10.5 \%(22 / 210)$. Of the 22 women with a diagnosis of deep vein thromboses, the left leg was involved in $18(82 \%)$. The proportion of women with deep vein thrombosis according to pregnancy stage was: 4 (18.2\%) during the first trimester, $1(4.5 \%)$ during the second, $9(40.9 \%)$ during the third, and 8 (36.4\%) during the postpartum period. Deep vein thrombosis was diagnosed on the basis of a positive compression ultrasonography test result in all but one woman. In this woman the diagnosis was made on the basis of the absence of Doppler flow in the iliac vein, with a "sludge" aspect in the common femoral vein. The diagnosis was formally confirmed two days later by a positive test result on repeated compression ultrasonography, with visualised endoluminal material.

The proportion of women with confirmed deep vein thrombosis differed significantly according to the trimester, the suspected affected leg, and the pretest clinical probability group (table $2 \Downarrow$ ). Deep vein thrombosis was confirmed in $16 \%$ of women in whom it was suspected in the left leg and $4.5 \%$ in whom it was suspected in the right, and in no women with bilateral symptoms. The association between empirical assessment of clinical probability and the proportion of women with confirmed deep vein thrombosis was strong: $2 / 107(1.9 \%)$ in the low probability group, $7 / 85(8.2 \%)$ in the intermediate group, and 13/18 (72.2\%) in the high group $(\mathrm{P}<0.001)$.

\section{Other ultrasonographic findings}

The ileocaval junction could not be imaged in 88 (41.9\%) women. Moreover, common iliac veins could not be imaged in $8(3.8 \%)$, all iliac veins in $9(4.3 \%)$, and popliteal veins in 1 $(0.5 \%)$. Among the 188 women in whom the diagnosis of deep vein thrombosis was excluded, an alternative diagnosis was made by ultrasonography in 26: 21 had isolated superficial thrombophlebitis, two had popliteal cysts, and one each had muscular tear, tendinitis, and painful inguinal adenopathy.

\section{Follow-up}

Of the 188 women with no deep vein thrombosis at initial examination, 10 received six weeks or more of anticoagulant treatment in the therapeutic range, mainly for the treatment of a superficial thrombophlebitis diagnosed at initial compression ultrasonography $(n=8)$. One woman was started on anticoagulant therapy by her doctor because of the discovery of a persistent foramen ovale, and another because of a history of two previous deep vein thromboses in the presence of antiphospholipid antibodies. These 10 women were excluded from further analysis. Three other patients received anticoagulation of shorter duration for the treatment of a superficial thrombophlebitis (7, 8 , and 10 days) and were kept in the main analysis. One woman was lost to follow-up. No patient died during follow-up. Thus, 177 women were available for analysis. During follow-up, no systematic serial compression ultrasonography was scheduled. Seven patients had diagnostic tests ordered by their treating doctors for clinically suspected venous thromboembolism. Six women had suspected deep vein thrombosis (all ipsilateral to the initial suspicion) and one had suspected pulmonary embolism. All these seven women underwent new compression ultrasonography and the patient with suspected pulmonary embolism also underwent a ventilation-perfusion lung scan. In four women with a suspected deep vein thrombosis, the compression ultrasonography result was negative, the women were left untreated, and a further three month follow-up was uneventful. The patient with suspected pulmonary embolism was admitted 50 days after the initial suspicion with fever and pleuritic chest pain. Both the compression ultrasonography and the ventilation-perfusion scan were interpreted as normal, and the final diagnosis was acute pneumonia. She received no anticoagulant therapy and further follow-up at three month was uneventful. These five women were adjudicated as having no recurrent venous thromboembolism. Two women had a positive compression ultrasonography result, showing proximal deep vein thrombosis during follow-up, and were adjudicated as having a confirmed proximal deep vein thrombosis. There was no disagreement between the two independent adjudicators. Thus the three month thromboembolic rate in patients left untreated on the basis of a negative single complete compression ultrasonography result was $2 / 177(1.1 \%, 95 \%$ confidence interval $0.3 \%$ to $4.0 \%)$, in pregnant women was $2 / 145(1.4 \%$, $0.4 \%$ to $4.9 \%)$ and in postpartum women was $0 / 32(0.0 \%, 0.0 \%$ to $10.7 \%)$. Table $3 \Downarrow$ summarises the medical history of the two women who experienced venous thromboembolic disease during follow-up.

\section{Discussion}

In this prospective management study, we included 226 pregnant and postpartum women with suspected lower limb deep vein thrombosis. We observed a $1.1 \%$ (95\% confidence interval $0.3 \%$ to $4.0 \%$ ) three month thromboembolic event rate in those left untreated on the basis of a negative single complete compression ultrasonography result. This result is in line with what was 
reported after a normal phlebography - the reference test-in non-pregnant patients ${ }^{3}$ and with the data obtained in a retrospective cohort study assessing the safety of a single complete compression ultrasonography in pregnant and postpartum women with suspected deep vein thrombosis. ${ }^{11}$

In venous thromboembolism management outcome studies, the occurrence of thromboembolic events during follow-up is considered as a failure of the initial diagnostic strategy. Admittedly, the $4 \%$ upper bound of the $95 \%$ confidence interval of the three month thromboembolic event rate in our study is slightly higher than the $3 \%$ limit proposed by some authors. ${ }^{14}$ Although this study is one of the largest available studies in the setting of pregnant and postpartum women, the sample size was relatively small, which could explain the wide confidence interval. The $4 \%$ upper bound we observed is in line with the results of a previous management outcome study in pregnant women, which reported a three month venous thromboembolis risk of 1/137 after negative serial proximal compression ultrasonography results - that is, a risk of $0.7 \%$ (95\% confidence interval $0.4 \%$ to $4.0 \%) .{ }^{15}$ However, we cannot exclude that diagnostic exclusion on the basis of a single complete compression ultrasonography result has a higher failure rate than diagnostic strategies for deep vein thrombosis in non-pregnant patients. If possible, larger studies should be conducted to provide narrower estimates of this failure rate. An increased risk of venous thromboembolic disease during pregnancy and the postpartum period could also partly account for the upper bound of our $95 \%$ confidence interval.

Nevertheless, it should be noted that among the two adjudicated events during follow-up, only one (patient A, table 3) clearly represents a failure of initial compression ultrasonography.

Patient B (table 3) could as well represent a de novo thrombotic episode. Indeed, this patient was admitted to hospital for pre-term labour. Fifteen days after admission, she presented with a swollen left leg and underwent her first compression ultrasonography. Ten days later she developed pre-eclampsia and underwent emergency caesarean section. She stayed immobilised in bed until postoperative day 5 , when she mentioned a novel leg pain and oedema. Repeated compression ultrasonography showed a proximal deep vein thrombosis.

Several other results deserve further discussion. Firstly, the prevalence of deep vein thrombosis in our study was $10.5 \%$, which is lower than usually observed in venous thromboembolic diagnostic studies in Europe. ${ }^{16}$ However, the proportion of confirmed venous thromboembolic disease is usually lower in pregnant women, which has been related to a lower threshold for clinical suspicion. ${ }^{15}{ }^{17}$ The lower prevalence, as well as the proportion of women with deep vein thrombosis in the left leg and the rate of confirmed deep vein thrombosis during the first trimester, are in line with a recent study. ${ }^{18}$ Also, we observed that almost $40 \%$ of deep vein thromboses occurred during the postpartum period, in line with another previous report. ${ }^{19}$

Secondly, most deep vein thromboses in our study were located in the proximal veins, as only two patients presented with isolated distal deep vein thrombosis. This may support the choice of a diagnostic strategy based on single complete compression ultrasonography in pregnant and postpartum women. Indeed, two recent prospective studies comparing single complete compression ultrasonography with serial proximal compression ultrasonography suggested that both strategies have similar safety in non-pregnant patients. ${ }^{20}{ }^{21}$ In these studies, performing single complete compression ultrasonography seemed more convenient since it avoided repeated compression ultrasonography, but at the expense of a significant increase in the proportion of patients in whom a distal deep vein thrombosis was diagnosed. This concern about over-diagnosis of deep vein thrombosis seems to be less relevant in pregnant and postpartum women because according to our data, only $10 \%$ of deep vein thromboses are distal in this population, compared with the 52\% reported in non-pregnant patients in a recent meta-analysis of diagnostic studies using complete compression ultrasonography. ${ }^{22}$

Thirdly, the diagnostic performance of clinical signs and symptoms is altered during pregnancy because pregnant women often experience symptoms compatible with deep vein thrombosis, and symptoms of deep vein thrombosis may vary during pregnancy. ${ }^{9}$ In our study, the assessment of clinical probability had no influence on the diagnostic strategy. Nevertheless, our data suggest that the empirical assessment of clinical probability remains accurate in this context. A strong association was found between empirical assessment of clinical probability and the proportion of confirmed deep vein thrombosis: $2 / 107(1.9 \%)$, in the low clinical probability class, $7 / 85(8.2 \%)$ in the intermediate probability class, and 13/18 $(72.2 \%)$, in the high clinical probability class. The two thromboembolic events during the follow-up occurred in patients not assessed as having a high clinical probability. Therefore, this stratification could not have been used to select patients for a more intensive diagnostic strategy. Anyhow, the lack of standardiz]sation and reproducibility of empirical assessment makes the validation and use of clinical prediction rules such as the recently derived LEFt (Left leg symptoms, Edema, First trimester presentation) score highly appealing. ${ }^{18}$

\section{Limitations of the study}

We chose to include pregnant women as well as postpartum women. This might be regarded as a limitation of our study. However, the technical limitations of ultrasound examination persist during the first days and weeks after delivery. For this reason it was important to check the safety of compression ultrasonography during this period. Noteably, most of our suspicions during the postpartum period occurred early: median 5 (interquartile range 3-26) days.

Current diagnostic strategies for suspected deep vein thrombosis in non-pregnant patients rely on the use of clinical probability and D-Dimer before the leg veins are imaged. We did not include this step in our investigations. Indeed, neither a management outcome study on the safety and usefulness of D-Dimer to rule out deep vein thrombosis in pregnant women, nor a validated clinical prediction rule in this setting, are available to date. Future studies may validate the use of a sequential diagnostic strategy in pregnant women with suspected deep vein thrombosis including the LEFt score, and the D-Dimer test, at the conventional $500 \mu \mathrm{g} / \mathrm{L}$ cut-off, or at a cut-off adjusted for pregnancy stage, as recently described. ${ }^{23}$

Finally, our study was a management outcome study. Admittedly this study design did not allow us to estimate either the exact number of patients with false negative and false positive results or the conventional accuracy indices (sensitivity, specificity, negative and positive predictive values) owing to the absence of a gold standard test. However, this study design is a crucial step in the validation of diagnostic strategies since it allows the clinical safety of a diagnostic strategy to be assessed. This design has been widely used in the past decades to validate all current diagnostic strategies for venous thromboembolic disease. ${ }^{24} 25$

\section{Conclusion}

The diagnosis of deep vein thrombosis during pregnancy is a diagnostic challenge. The low prevalence of deep vein 
thrombosis and the need for a non-radiating diagnostic strategy in these women render non-invasive diagnostic tools such as compression ultrasonography highly appealing. Our study shows that single complete compression ultrasonography might safely rule out the diagnosis of deep vein thrombosis in pregnant and postpartum women. However, the limitations described above as well as the upper limit of the $95 \%$ confidence interval around our estimate of the three month risk of a thromboembolic event prevent us from drawing firm conclusions. Further investigations should aim at confirming these results and evaluating the use of compression ultrasonography in a sequential diagnostic strategy including assessment of clinical probability and D-dimer measurement.

We thank the residents and physicians from the emergency departments, radiology, and ultrasonography units of all participating centres; the study nurses, secretaries, and clinical research associates for their invaluable help; the members of the adjudication committee: Françoise Boehlen and François Becker, for their important contribution; and the participants who made the study possible. The EDVIGE study group: Dominique Bernard (Carhaix), Jean Broussaud (Vannes), Paul Cirafici (Geneva), Cécile de Gail Sauteron (Saint-Grégoire), Philippe de Moerloose (Genève), Marie-Annick Direr (Brest), Catherine Duigou-Bihi (Lorient), Pierre Fontana (Geneva), Jacques Gestin (Quimper), Salah Gueddi (Geneva), Bruno Guias (Morlaix), Bruno Guichaoua (Quimper), Patrice Hudo (Saint-Brieuc), Nathalie Journeaux (Rennes), Raymond Kaczmarek (Quimper), Dominique le Berre (Paimpol), Isabelle Leborgne (Rennes), Marie-Pierre le Goc Pedelucq (Lorient), Dominique le Menn Bureau (Pontivy), Fabrice le Thoer (Brest), Stéphanie Louis (Brest), Geneviève Madec Bougeard (Lannion), Mario Maufus (Grenoble), Catherine Noel Morel (Pacé), Karine Pichon (Vannes), Emmanuel Plat (Brest), Laurent Pruvost (Saint-Malo), Philippe Quehe (Brest), Edith Rivoal (Quimper), Eric Thulliez (Rennes), Johny Vogel (Sierre), and Robert Wutschert (Neuchatel).

Contributors: GLG and MR coordinated the study, analysed the data, and drafted the paper. They are the guarantors. HB and DM provided administrative support. All other authors participated in the study design, data collection, and critical revision of the manuscript, and approved the final version. All the members of the EDVIGE study group designed the study and collected data for the study.

Funding: The study was supported by grants from the Projet Hospitalier de Recherche Clinique (grant No 2005 R 08.01) in France, and from the Swiss National Foundation (grant No 3200B0-120760) in Switzerland. The sponsors had no role in the study design and the collection, analysis, and interpretation of data, the writing of the article, and the decision to submit it for publication.

Competing interests: All authors have completed the ICMJE uniform disclosure form at www.icmje.org/coi_disclosure.pdf (available on request from the corresponding author) and declare: no support from any organisation for the submitted work; no financial relationships with any organisations that might have an interest in the submitted work in the previous three years; and no other relationships or activities that could appear to have influenced the submitted work.

Ethical approval: This study was approved by the ethics committees of the Commission centrale d'éthique de la recherche sur l'être humain, Geneva University Hospital, Geneva, Switzerland, and Comité d'Ethique du Centre Hospitalier Universitaire de Brest, Brest, France.

Data sharing: No additional data available.
1 Hull RD, Raskob GE, Carter CJ. Serial impedance plethysmography in pregnant patients with clinically suspected deep-vein thrombosis. Clinical validity of negative findings. Ann Intern Med 1990;112:663-7.

2 Chan WS, Ginsberg JS. Diagnosis of venous thromboembolism in pregnancy: a study in extrapolation or a science in evolution? Expert Rev Cardiovasc Ther 2009;7:1479-82.

3 Hull R, Hirsh J, Sackett DL, Taylor DW, Carter C, Turpie AG, et al. Clinical validity of a negative venogram in patients with clinically suspected venous thrombosis. Circulation 1981;64:622-5.

4 Heijboer H, Buller HR, Lensing AW, Turpie AG, Colly LP, ten Cate JW. A comparison of real-time compression ultrasonography with impedance plethysmography for the diagnosis of deep-vein thrombosis in symptomatic outpatients. N Engl J Med 1993;329:1365-9.

5 Righini M, Perrier A, De Moerloose P, Bounameaux H. D-Dimer for venous thromboembolism diagnosis: 20 years later. J Thromb Haemost 2008:6:1059-71.

6 Kearon C, Ginsberg JS, Hirsh J. The role of venous ultrasonography in the diagnosis of suspected deep venous thrombosis and pulmonary embolism. Ann Intern Med 1998;129:1044-9.

7 Stevens SM, Elliott CG, Chan KJ, Egger MJ, Ahmed KM. Withholding anticoagulation after a negative result on duplex ultrasonography for suspected symptomatic deep venous thrombosis. Ann Intern Med 2004;140:985-91.

8 Righini M. Is it worth diagnosing and treating distal deep vein thrombosis? No. J Thromb Haemost 2007;5(Suppl 1):55-9.

9 Greer IA. Thrombosis in pregnancy: maternal and fetal issues. Lancet 1999;353:1258-65.

10 Melis F, Vandenbrouke JP, Buller HR, Colly LP, Bloemenkamp KW. Estimates of risk of venous thrombosis during pregnancy and puerperium are not influenced by diagnostic suspicion and referral basis. Am J Obstet Gynecol 2004;191:825-9.

11 Le Gal G, Prins AM, Righini M, Bohec C, Lacut K, Germain P, et al. Diagnostic value of a negative single complete compression ultrasound of the lower limbs to exclude the diagnosis of deep venous thrombosis in pregnant or postpartum women: a retrospective hospital-based study. Thromb Res 2006;118:691-7.

12 Schellong SM, Beyer J, Kakkar AK, Halbritter K, Eriksson BI, Turpie AG, et al. Ultrasound screening for asymptomatic deep vein thrombosis after major orthopaedic surgery: the VENUS study. J Thromb Haemost 2007:5:1431-7.

13 Righini M, Le Gal G, Aujesky D, Roy PM, Sanchez O, Verschuren F, et al. Complete venous ultrasound in outpatients with suspected pulmonary embolism. J Thromb Haemost 2009;7:406-12

14 Kruip MJ, Leclercq MG, van der Heul C, Prins MH, Buller HR. Diagnostic strategies for excluding pulmonary embolism in clinical outcome studies. A systematic review. Ann Intern Med 2003;138:941-51.

15 Chan WS, Chunilal S, Lee A, Crowther M, Rodger M, Ginsberg JS. A red blood cell agglutination D-dimer test to exclude deep venous thrombosis in pregnancy. Ann Intern Med 2007;147:165-70.

16 Le Gal G, Bounameaux H. Diagnosing pulmonary embolism: running after the decreasing prevalence of cases among suspected patients. J Thromb Haemost 2004;2:1244-6.

17 Chan WS, Ray JG, Murray S, Coady GE, Coates G, Ginsberg JS. Suspected pulmonary embolism in pregnancy: clinical presentation, results of lung scanning, and subsequent maternal and pediatric outcomes. Arch Intern Med 2002;162:1170-5.

18 Chan WS, Lee A, Spencer FA, Crowther M, Rodger M, Ramsay T, et al. Predicting deep venous thrombosis in pregnancy: out in "LEFt" field? Ann Intern Med 2009;151:85-92.

19 Jacobsen AF, Skjeldestad FE, Sandset PM. Incidence and risk patterns of venous thromboembolism in pregnancy and puerperium - a register-based case-control study. Am J Obstet Gynecol 2008:198:233 e1-7.

20 Bernardi E, Camporese G, Buller HR, Siragusa S, Imberti D, Berchio A, et al. Serial 2-point ultrasonography plus D-dimer vs whole-leg color-coded Doppler ultrasonography for diagnosing suspected symptomatic deep vein thrombosis: a randomized controlled trial. JAMA 2008;300:1653-9.

21 Gibson NS, Schellong SM, Kheir DY, Beyer-Westendorf J, Gallus AS, McRae S, et al. Safety and sensitivity of two ultrasound strategies in patients with clinically suspected deep venous thrombosis: a prospective management study. J Thromb Haemost 2009;7:2035-41.

22 Johnson SA, Stevens SM, Woller SC, Lake E, Donadini M, Cheng J, et al. Risk of deep vein thrombosis following a single negative whole-leg compression ultrasound: a systematic review and meta-analysis. JAMA 2010;303:438-45.

23 Chan WS, Lee A, Spencer FA, Chunilal S, Crowther M, Wu W, et al. D-dimer testing in pregnant patients: towards determining the next 'level' in the diagnosis of deep vein thrombosis. J Thromb Haemost 2010;8:1004-11.

24 Righini M, Le Gal G, Aujesky D, Roy PM, Sanchez O, Verschuren F, et al. Diagnosis of pulmonary embolism by multidetector $\mathrm{CT}$ alone or combined with venous ultrasonography of the leg: a randomised non-inferiority trial. Lancet 2008;371:1343-52.

25 Van Belle A, Buller HR, Huisman MV, Huisman PM, Kaasjager K, Kamphuisen PW, et al. Effectiveness of managing suspected pulmonary embolism using an algorithm combining clinical probability, D-dimer testing, and computed tomography. JAMA 2006;295:172-9.

Accepted: 12 March 2012

\section{Cite this as: BMJ 2012:344:e2635}

This is an open-access article distributed under the terms of the Creative Commons Attribution Non-commercial License, which permits use, distribution, and reproduction in any medium, provided the original work is properly cited, the use is non commercial and is otherwise in compliance with the license. See: http://creativecommons.org/licenses/bync/2.0/ and http://creativecommons.org/licenses/by-nc/2.0/legalcode. 


\section{What is already known on this topic}

Single complete compression ultrasonography is widely used to rule out deep vein thrombosis in everyday clinical practice No data are available to support this finding in the setting of pregnancy and the postpartum period

\section{What this study adds}

Single complete compression ultrasonography may safely rule out deep vein thrombosis in pregnant and postpartum women Of 177 women without deep vein thrombosis and who did not receive full dose anticoagulant therapy, two experienced an objectively confirmed deep vein thrombosis during follow-up

\section{Tables}

\begin{tabular}{lc}
\hline Table 1 | General characteristics of pregnant and postpartum women with suspected deep vein thrombosis. Values are numbers (percentages) \\
unless stated otherwise & Total \\
Characteristics & $33(28-37)$ \\
\hline Age (interquartile range) years & $24.3(21.5-27.5)$ \\
\hline Body mass index (interquartile range) & $9(5-13)$ \\
\hline Weight gain (interquartile range) kg & $20(11.0)$ \\
\hline Stage of pregnancy: & $51(24.3)$ \\
\hline First trimester & $96(45.7)$ \\
\hline Second trimester & $43(20.5)$ \\
\hline Third trimester & $26(12.4)$ \\
\hline Post partum & $46(21.9)$ \\
\hline Risk factors: & $10(4.8)$ \\
\hline Personal history of VTE & $25(11.9)$ \\
\hline Family history of VTE & $6(2.9)$ \\
\hline Known thrombophilia & $57(27.1)$ \\
\hline Recent immobilisation & $17(8.1)$ \\
\hline Recent surgery or trauma & $6(2.9)$ \\
\hline Varicose veins & $12(5.7)$ \\
\hline Complicated pregnancy* & \\
\hline Twin pregnancy & \\
\hline Recent travel (>6 hours) & \\
\hline
\end{tabular}

VTE=venous thromboembolism.

*Encompassed gestational diabetes $(n=6)$, preterm labour $(n=4)$, intrauterine growth restriction $(n=2)$, pre-eclampsia $(n=2)$, placenta praevia $(n=1)$, and ovarian hyperstimulation syndrome $(n=1)$. 
Table 2| Proportion of women with confirmed deep vein thrombosis according to pregnancy trimester, suspected affected leg, and clinical probability of having deep vein thrombosis

\begin{tabular}{|c|c|c|}
\hline Variables & No with deep vein thrombosis/No in group (\%) & P value* \\
\hline \multicolumn{3}{|c|}{ Pregnancy trimester: } \\
\hline First & $4 / 20(20.0)$ & 0.03 \\
\hline Second & $1 / 51(2.0)$ & \\
\hline Third & $9 / 96(9.4)$ & \\
\hline Post partum & $8 / 43(18.6)$ & \\
\hline \multicolumn{3}{|l|}{ Suspected leg: } \\
\hline Right & $4 / 88(4.5)$ & 0.02 \\
\hline Left & $18 / 112(16.1)$ & \\
\hline Both legs & $0 / 10(0.0)$ & \\
\hline \multicolumn{3}{|c|}{ Clinical probability: } \\
\hline Low & 2/107 (1.9) & $<0.001$ \\
\hline Intermediate & $7 / 85(8.2)$ & \\
\hline High & $13 / 18(72.2)$ & \\
\hline
\end{tabular}


Table 3| Summary of medical history of two women adjudicated as having proximal deep vein thrombosis during three month follow-up

\begin{tabular}{|c|c|c|}
\hline Variables & Patient A & Patient B \\
\hline Age (years) & 25 & 23 \\
\hline Body mass index & 27.3 & 21.9 \\
\hline Stage of pregnancy (weeks) & 34 & 29 \\
\hline Risk factors & 0 & 0 \\
\hline \multicolumn{3}{|l|}{ Clinical probability of deep vein thrombosis: } \\
\hline \multicolumn{3}{|l|}{ Low } \\
\hline Intermediate & Yes & Yes \\
\hline \multicolumn{3}{|l|}{ High } \\
\hline \multicolumn{3}{|l|}{ Initial compression ultrasonography: } \\
\hline All venous segments imaged & Yes & Yes \\
\hline Ileocaval junction imaged & Yes & Yes \\
\hline Respiratory modulation of iliac blood flow & Yes & No \\
\hline Compression ultrasonography result & Normal & Normal \\
\hline \multicolumn{3}{|l|}{ Compression ultrasonography during follow-up: } \\
\hline Date & Day 1 & Day 15 \\
\hline Result & Left iliofemoral deep vein thrombosis & Left iliofemoral deep vein thrombosis \\
\hline Comments & $\begin{array}{l}\text { Returned to emergency unit next day because of } \\
\text { increasing symptoms. Compression ultrasonography } \\
\text { result was clearly positive for deep vein thrombosis }\end{array}$ & $\begin{array}{l}\text { Was admitted for premature labour } 15 \text { days before initial } \\
\text { suspicion. Emergency caesarean section carried out five } \\
\text { days after initial suspicion. Suspected deep vein thrombosis } \\
\text { confirmed by compression ultrasonography on postoperative } \\
\text { day } 5\end{array}$ \\
\hline
\end{tabular}




\section{Figure}

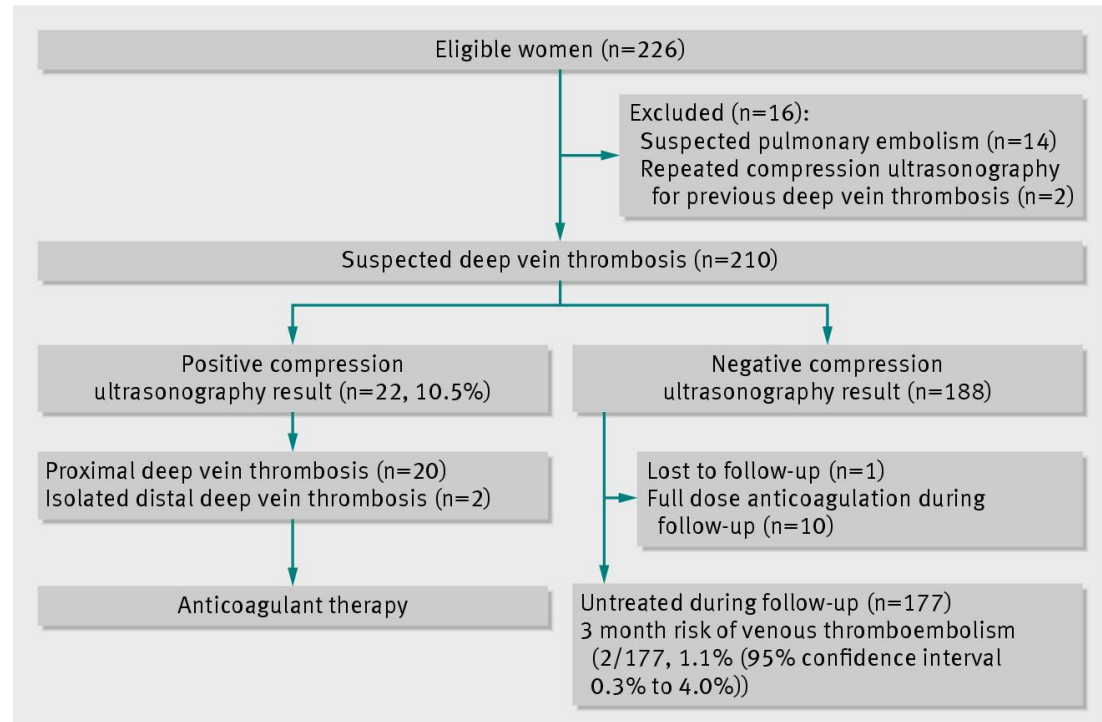

Flow of participants through study 